\title{
A Linear Three-Level Difference Scheme for Quasilinear Parabolic Equations*
}

\section{By Milton Lees}

The purpose of this paper is to establish a convergence theorem for a second order accurate, linear, three-level difference scheme for a class of quasilinear parabolic differential equations. In the interests of notational simplicity, we shall present our result in detail only for the special semilinear partial differential equation

$$
b(w) \frac{\partial w}{\partial t}=\frac{\partial}{\partial x}\left(a(w) \frac{\partial w}{\partial x}\right) .
$$

Later, we shall indicate how our convergence theorem can be extended to more general equations than (1a).

Let $w=w(x, t)$ be a smooth solution of the differential equation in the rectangular region $R=\{(x, t): 0 \leqq x \leqq 1,0 \leqq t \leqq T\}$ such that

$$
\begin{array}{ll}
w(x, 0)=\phi(x), & 0 \leqq x \leqq 1, \\
w(0, t)=\psi_{0}(t), & 0 \leqq t \leqq T, \\
w(1, t)=\psi_{1}(t), & 0 \leqq t \leqq T,
\end{array}
$$

where $\phi, \psi_{0}$ and $\psi_{1}$ are specified functions. In addition to assuming that the coefficient functions $b$ and $a$ are defined and smooth for all real $w$, we assume that there exist positive constants $\mu$ and $\eta$ such that

$$
b(w) \geqq \mu>0 \quad \text { and } \quad a(w) \geqq \eta>0 .
$$

Because of (2), it follows that the differential equation (1a) is uniformly parabolic, so that $w$ is the only solution of the boundary value problem (1).

Before describing the three-level difference scheme for (1), we introduce some convenient notation. If $N$ is a positive integer and $h>0$ is defined by the relation $(N+1) h=1$, then we put $X=\{s h: s=0,1, \cdots, N+1\}$. If $0<2 k<T$, then we let $J$ be the largest positive integer such that $k J \leqq T$. We assume that there are two positive constants $\lambda_{-}$and $\lambda_{+}$such that all admissible $h$ and $k$ satisfy

$$
\lambda_{-} h \leqq k \leqq \lambda_{+} h \text {. }
$$

The standard difference operators $D_{+}, D_{-}$and $D_{0}$ are defined, as follows:

$$
\begin{aligned}
h D_{+} u(x) & =u(x+h)-u(x), \\
h D_{-} u(x) & =u(x)-u(x-h), \\
2 h D_{0} u(x) & =u(x+h)-u(x-h) .
\end{aligned}
$$

Finally, for any suitably defined function $v(x)$, we put

$$
\bar{a}(v(x))=a\{[v(x)+v(x-h)] / 2\} .
$$

Received April 27, 1966.

* This research was supported in part by NSF Grant GP-5553. 
We determine a sequence $u_{0}, u_{1}, \cdots, u_{j}$ of real valued functions defined on the $(N+2)$-element set $X$, as follows: For each $j$ we put $u_{j}(0)=\psi_{0}(j k)$ and $u_{j}(1)=\psi_{1}(j k)$. Then, on the $N$-element set $X \cap(0,1)$,

$$
\begin{aligned}
& u_{0}=\phi, \\
& u_{1}=\phi+\frac{k}{b(\phi)}\left(a(\phi) \phi^{\prime}\right)^{\prime},
\end{aligned}
$$

and, for $1 \leqq j<J$,

$$
b\left(u_{j}\right)\left(u_{j+1}-u_{j-1}\right)=2 k D_{+}\left(\bar{a}\left(u_{j}\right) D_{-} \hat{u}_{j}\right),
$$

where

$$
\hat{u}_{j}=\left(u_{j+1}+u_{j}+u_{j-1}\right) / 3 .
$$

It is clear that the three-level difference equation (4c) determines $u_{j+1}$ uniquely as the solution of a linear, numerically stable, tridiagonal system of equations. The relations (4a) and (4b) provide the necessary starting values for (4c). Although we give no details, it will become obvious that it is permissible to replace (4b) by

$$
u_{1}=\phi+\frac{k}{b(\phi)}\left[D_{+}\left(\bar{a}(\phi) D_{-} \phi\right)\right] \text {. }
$$

For the approximation determined by (5) we have the convergence theorem.

Theorem. There is a constant $A>0$, independent of $h, k$ and $u_{j}$ such that

$$
\max _{x \in X ; 1 \leqq j \leqq J}\left|u_{j}(x)-w(x, j k)\right| \leqq A\left(h^{2}+k^{2}\right)
$$

for all sufficiently small $h$ and $k$ satisfying (3).

Previously, the weaker error estimate

$$
\max _{1 \leqq j \leqq J}\left(h \sum_{s=1}^{N}\left|u_{j}(s h)-w(s h, j k)\right|^{2}\right)^{1 / 2} \leqq A\left(h^{2}+k^{2}\right)
$$

was proved for certain mild (linear) generalizations of the heat equation [1], [3]. Also, in a private communication, James Gunn showed us a derivation of the error estimate ( 7 ) for equations of the form

$$
b(x, t) \frac{\partial w}{\partial t}=\frac{\partial}{\partial x}\left(a(x, t, w) \frac{\partial w}{\partial x}\right)+c(x, t, w) .
$$

His proof, however, assumes, in addition to the usual parabolicity inequalities, that $\partial c / \partial w$ is bounded for $(x, t) \in R$ and $|w|<+\infty$ and $a$ has continuous second and bounded third derivatives in the same region.

Let us denote by $\Omega_{h}$ the real linear space of all real-valued functions $u(x)$ defined on the discrete set $X$ for which $u(0)=u(1)=0$. It is obvious that $\Omega_{h}$ has dimension $N$. The $l_{2}$-inner product $(u, v)$ and induced norm $\|u\|=(u, v)^{1 / 2}$ on $\Omega_{h}$ are defined by

$$
(u, v)=h \sum_{s=1}^{N} u(s h) v(s h) .
$$

The $l_{\infty}$-norm $\|u\|_{\infty}$ on $\Omega_{h}$ is given by

$$
\|u\|_{\infty}=\max _{1 \leqq s \leqq N}|u(s h)| .
$$


The $D$-inner product $(u, v)_{D}$ and induced $D$-norm $\|u\|_{D}=(u, v)_{D}{ }^{1 / 2}$ on $\Omega_{h}$ are defined by

$$
(u, v)_{D}=h \sum_{s=1}^{N+1} D \_u(s h) D \_v(s h) .
$$

It is well known [2] that the foregoing norms satisfy the inequalities

$$
\|u\| \leqq\|u\|_{\infty} \leqq\|u\|_{D} .
$$

For the solution $u$ of $(1)$ we put $w_{j}(x)=w(x, j k)$. We define on $\Omega_{h}$ a modified $D$-inner product $(u, v)_{j, D}$ and induced norm $\|u\|_{j, D}=(u, v)_{j, D}^{1 / 2}$ by

$$
(u, v)_{j, D}=h \sum_{s=1}^{N+1} \bar{a}\left(u_{j}(s h)\right) D \_u(s h) D \_v(s h) .
$$

It follows immediately from (2) that

$$
\|u\|_{j, D} \geqq \eta^{1 / 2}\|u\|_{D}, \quad 0 \leqq j \leqq J
$$

Furthermore, if

$$
A_{1}=\sup _{R}\left|\frac{\partial w}{\partial t}\right| \sup _{R}\left|a^{\prime}(w(x, t))\right|,
$$

then from the mean value theorem and (9) we obtain the inequalities

$$
\begin{aligned}
\left|(u, v)_{j, D}-(u, v)_{j-1, D}\right| & \leqq k A_{1}\|u\|_{D}\|v\|_{D}, \\
\|u\|_{j, D}^{2} & \leqq\left(1+k \eta^{-1} A_{1}\right)\|u\|_{j-1, D}^{2} .
\end{aligned}
$$

Finally, summation by parts shows that [2]

$$
\left(u, D_{+}\left(\bar{a}\left(u_{j}\right) D \_v\right)\right)=-(u, v)_{j, D}
$$

for any $u, v \in \Omega_{h}$.

With the aid of these preliminary results, we proceed to the proof of the convergence theorem. The usual argument, based on Taylor's formula, shows that the solution $w$ of (1) satisfies, in $X \cap(0,1)$, the relations:

$$
\begin{aligned}
& w_{0}=\phi, \\
& w_{1}=u_{1}+\tau_{1},
\end{aligned}
$$

and, for $1 \leqq j<J$,

$$
b\left(w_{j}\right)\left(u_{j+1}-u_{j-1}\right)=2 k D_{+}\left(\bar{a}\left(w_{j}\right) D_{-} \hat{w}_{j}\right)+2 k \tau_{j+1},
$$

where $\hat{w}_{j}$ is defined as in (5) and the local truncation errors $\tau_{j}$ are such that

$$
\left\|\tau_{j}\right\|_{\infty} \leqq A_{2}\left(h^{2}+k^{2}\right), \quad 1 \leqq j \leqq J,
$$

for a suitable constant $A_{2}$. Without loss of generality, we may assume also that

$$
\left\|w_{1}-u_{1}\right\|_{D} \leqq A_{2}\left(h^{2}+k^{2}\right) \text {. }
$$

Obviously, the error function $z_{j}=u_{j}-u_{j}$ belongs to the space $\Omega_{h}$, and it 
follows from (4), (13) and the mean value theorem that

$$
\begin{aligned}
& z_{0}=0, \\
& z_{1}=\tau_{1},
\end{aligned}
$$

and, for $1 \leqq j<J$,

$$
\begin{aligned}
b\left(u_{j}\right)\left(z_{j+1}-z_{j-1}\right)= & 2 k D_{+}\left(\bar{a}\left(w_{j}\right) D_{-} \hat{z}_{j}\right)+2 k \tau_{j+1} \\
& +b^{\prime}\left(\Theta_{j}\right) z_{j}\left(w_{j+1}-w_{j-1}\right) \\
& +2 k D_{+}\left(\sigma_{j} D_{-} \hat{w}_{j}\right)-2 k D_{+}\left(\sigma_{j} D_{-} \hat{z}_{j}\right),
\end{aligned}
$$

where

$$
\sigma_{j}(x)=\bar{a}\left(w_{j}(x)\right)-\bar{a}\left(u_{j}(x)\right), \quad x \in X-\{0\},
$$

and

$$
\left|\Theta_{j}\right| \leqq \sup _{R}|w|+\left\|z_{j}\right\|_{\infty} .
$$

First, we choose $h$ and $k$ so small that

$$
A_{2}\left(1+\lambda_{-}^{-2}\right) k^{2} \leqq k \leqq 1 .
$$

Then, using (3), (8) and (15) we find that

$$
\left\|z_{1}\right\|_{\infty} \leqq\left\|z_{1}\right\|_{D} \leqq k \leqq 1 .
$$

Now, we prove by induction that $\left\|z_{j}\right\|_{\infty} \leqq k$ for all sufficiently small $h$ and $k$ satisfying (3). It follows from (16a) and (19) that the assertion is true for $j=0$ and $j=1$. Assume that $\left\|z_{l}\right\|_{\infty} \leqq k$ for all nonnegative integers $l \leqq j$, and we shall prove that $\left\|z_{j+1}\right\|_{\infty} \leqq k$.

Putting $z_{j}{ }^{*}=z_{j+1}-z_{j-1}$, we form the $l_{2}$-inner product of (16c) with $3 z_{j}{ }^{*}$ to obtain

$$
I_{1}+I_{2}=\sum_{r=3}^{6} I_{r}
$$

where

$$
\begin{aligned}
& I_{1}=3\left(z_{j}{ }^{*}, b\left(u_{j}\right) z_{j}{ }^{*}\right), \\
& I_{2}=-6 k\left(z_{j}^{*}, D_{+}\left(\bar{a}\left(w_{j}\right) D_{-} \hat{z}_{j}\right)\right), \\
& I_{3}=6 k\left(z_{j}{ }^{*}, \tau_{j+1}\right), \\
& I_{4}=3\left(z_{j}{ }^{*}, b^{\prime}\left(\Theta_{j}\right) z_{j}\left(w_{j+1}-w_{j-1}\right\}\right), \\
& I_{5}=6 k\left(z_{j}{ }^{*}, D_{+}\left(\sigma_{j} D_{-} \hat{w}_{j}\right)\right), \\
& I_{6}=-6 k\left(z_{j}{ }^{*}, D_{+}\left(\sigma_{j} D_{-} \hat{z}_{j}\right)\right) .
\end{aligned}
$$

It follows immediately from (2) that

$$
I_{1} \geqq 3 \mu\left\|z_{j}^{*}\right\|^{2} \text {. }
$$


Using (5) and (12) we obtain the relation

$$
I_{2}=2 k\left[\left\|z_{j+1}\right\|_{i, D}^{2}+\left(z_{j+1}, z_{j}\right)_{j, D}-\left\|z_{j-1}\right\|_{j, D}^{2}-\left(z_{j}, z_{j-1}\right)_{j, D}\right] .
$$

If we put

$$
L_{j}=2\left[\left\|z_{j+1}\right\|_{j, D}^{2}+\left(z_{j+1}, z_{j}\right)_{j, D}+\left\|z_{j}\right\|_{j, D}^{2}\right]
$$

then

$$
I_{2}=k L_{j}-2 k\left[\left\|z_{j}\right\|_{j, D}^{2}+\left(z_{j}, z_{j-1}\right)_{j, D}+\left\|z_{j-1}\right\|_{j, D}^{2}\right] .
$$

Using (10), (11) and Schwarz's inequality we obtain the estimate

$$
I_{2} \geqq k L_{j}-k\left(1+k A_{3}\right) L_{j-1},
$$

where

$$
A_{3}=3 \eta^{-1} A_{1} \text {. }
$$

Using the generalized Cauchy-Schwarz inequality on $I_{3}$ we find that

$$
\left|I_{3}\right| \leqq \epsilon\left\|z_{j}^{*}\right\|^{2}+9 k^{2} \epsilon^{-1}\left\|\tau_{j+1}\right\|^{2}
$$

for any positive number $\epsilon$.

Our induction assumption $\left\|z_{j}\right\|_{\infty} \leqq k \leqq 1$ and (18) imply that $\left|\Theta_{j}\right| \leqq m+1$, where $m=\sup _{R}|w|$; hence, if

$$
A_{4}=\frac{3}{2} \sup _{|w| \leqq m+1}\left|b^{\prime}(w)\right| \sup _{R}\left|\frac{\partial w}{\partial t}\right|
$$

then

$$
\left|I_{4}\right| \leqq 2 k A_{4}\left\|z_{j}^{*}\right\|\left\|z_{j}\right\|
$$

Therefore, by (8)

$$
\left|I_{4}\right| \leqq \epsilon\left\|z_{j}^{*}\right\|^{2}+k^{2} A_{4}{ }^{2} \epsilon^{-1}\left\|z_{j}\right\|_{D}^{2} .
$$

Since our induction assumption implies that $\left\|u_{j}\right\|_{\infty} \leqq\left\|w_{j}\right\|_{\infty}+\left\|z_{j}\right\|_{\infty} \leqq m+1$, using (17) we can find a constant $A_{5}>0$, depending only on $\lambda_{+}, \sup _{R}|\partial w / \partial x|$, $\sup _{R}\left|\partial^{2} w / \partial x^{2}\right|$, and

$$
\sup _{|w| \leqq m+1}\left[\left|a^{\prime}(w)\right|,\left|a^{\prime \prime}(w)\right|\right]
$$

such that

$$
\left\|D_{+}\left(\sigma_{j} D_{-} \hat{w}_{j}\right)\right\| \leqq A_{5}\left\|z_{j}\right\|_{D}
$$

hence, for any $\epsilon>0$, we have the estimate

$$
\left|I_{5}\right| \leqq \epsilon\left\|z_{j}^{*}\right\|^{2}+9 k^{2} \epsilon^{-1} A_{5}^{2}\left\|z_{j}\right\|_{D}^{2} .
$$

Finally, if

$$
A_{6}=3 \eta^{-1} \sup _{|w| \leqq m+1}\left|a^{\prime}(w)\right|
$$

then, using summation by parts and Schwarz's inequality, we find that

$$
\left|I_{6}\right| \leqq k^{2} A_{6}\left(L_{j}+L_{-1}\right) \text {. }
$$


Now, from (20) and (21) we obtain the estimate

$$
\begin{aligned}
(3 \mu-3 \epsilon)\left\|z_{j}^{*}\right\|^{2}+k\left(1-k A_{6}\right) L_{j} \leqq & k\left(1+k A_{7}\right) L_{j-1} \\
& +9 k^{2} \epsilon^{-1}\left\|\tau_{j+1}\right\|^{2}+k^{2} A_{8} \epsilon^{-1}\left\|z_{j}\right\|_{D}^{2},
\end{aligned}
$$

where

$$
A_{7}=A_{3}+A_{6} \text { and } A_{8}=A_{4}{ }^{2}+9 A_{5}{ }^{2} .
$$

Choose $\epsilon=\mu$. Then (22) implies that

$$
\left(1-k A_{6}\right) L_{j} \leqq\left(1+k A_{9}\right) L_{j-1}+9 k \mu^{-1}\left\|\tau_{j+1}\right\|^{2},
$$

where

$$
A_{9}=A_{7}+\mu^{-1} \eta^{-1} A_{7} .
$$

We now assume $k$ is so small that $2 k A_{6} \leqq 1$. Since

$$
\left(1-k A_{6}\right)^{-1} \leqq \exp \left(2 k A_{6}\right) \text {, }
$$

it follows from (23) that

$$
L_{j} \leqq e^{\alpha k} L_{j-1}+9 k \mu^{-1}\left\|\tau_{j+1}\right\|^{2},
$$

where

$$
\alpha=2 A_{6}+A_{9} .
$$

If $\beta=9 \mu^{-1} A_{2}^{2} T$, then (14) and (24) imply that

$$
L_{j} \leqq e^{\alpha T}\left[L_{0}+\beta\left(h^{2}+k^{2}\right)^{2}\right] .
$$

Since $L_{0}=2\left\|z_{1}\right\|_{0, D}^{2} \leqq 2 \eta^{-1} A_{2}{ }^{2}\left(h^{2}+k^{2}\right)^{2}$, in view of (9) and (15), we obtain the inequality

$$
\left\|z_{j+1}\right\|_{\infty}^{2} \leqq\left\|z_{j+1}\right\|_{D}^{2} \leqq \eta^{-1}\left\|z_{j+1}\right\|_{j, D}^{2} \leqq 4 \eta^{-1} L_{j} \leqq A^{2}\left(h^{2}+k^{2}\right)^{2}
$$

where

$$
A^{2}=8 \eta^{-1} e^{\alpha T}\left(A_{2}^{2}+\beta\right) .
$$

Thus, we shall have $\left\|\boldsymbol{z}_{j+1}\right\|_{\infty} \leqq k$ as soon as

$$
A\left(1+\lambda_{-}{ }^{-2}\right) k \leqq 1,
$$

which finishes the induction step (note that our restrictions on the size of $h$ and $k$ are independent of $u_{j}$ ).

As a result of (25), we have also established the convergence theorem: $\left\|z_{j}\right\|_{\infty} \leqq$ $A\left(h^{2}+k^{2}\right)$.

An examination of the foregoing proof will show that the convergence theorem remains valid under the weaker assumption that the conditions in (2) hold only in some neighborhood of the solution $w$. This generalization shows, for example, that the convergence theorem holds when the difference scheme (4) is applied to positive solutions of

$$
w^{1 / 2} \partial w / \partial t=\partial^{2} w / \partial x^{2}
$$


Finally, we indicate that our results can be extended to equations of the form

$$
b\left(x, t, w, \frac{\partial w}{\partial x}\right) \frac{\partial w}{\partial t}=\frac{\partial}{\partial x}\left(a(x, t, w) \frac{\partial w}{\partial x}\right)+c\left(x, t, w, \frac{\partial w}{\partial x}\right),
$$

where $b \geqq \mu>0$ and $a \geqq \eta>0$. We put

$$
\bar{a}(x, t v(x))=a\left[x-\frac{h}{2}, t, \frac{v(x)+v(x-h)}{2}\right] .
$$

Then the difference scheme becomes $u_{0}=\phi$,

$$
u_{1}=\phi+\left[k / b\left(x, 0, \phi, \phi^{\prime}\right)\right]\left[\left(a(x, 0, \phi) \phi^{\prime}\right)^{\prime}+f\left(x, 0, \phi, \phi^{\prime}\right)\right],
$$

and, for $1 \leqq j<J$

$b\left(x, j k, u_{j}, D_{0} u_{j}\right)\left(u_{j+1}-u_{j-1}\right)=2 k D_{+}\left(\bar{a}\left(x, j k, u_{j}\right) D_{-} \hat{u}_{j}\right)+2 k f\left(x, j k, u_{j}, D_{0} u_{j}\right)$.

The only major change in the convergence proof is that one proves by induction that $\left\|z_{j}\right\|_{\infty} \leqq k$ and

$$
\max _{x \rightarrow\{0\}}\left|D_{-} z_{j}\right| \leqq k
$$

Case Institute of Technology

Cleveland, Ohio

1. J. Douglas, JR., A Survey of Numerical Methods for Parabolic Differential Equations, Advances in Computers, Yol. 2, Academic Press, New York, 1961, pp. 1-52. MR 25 \#5604.

2. M. LeEs, "Approximate solution of parabolic equations," J. Soc. Indust. Appl. Math., v. 7, 1959, pp. 167-183. MR 22 \#1092.

3. R. D. Richtm Yer, Difference Methods for Initial-Value Problems, Interscience Tracts in Pure and Appl. Math., Tract 4, Interscience, New York, 1957. MP $20 \# 438$. 